\title{
LA GASTRONOMÍA COMO AGENTE MOTIVADOR DE VIAJE
}

\section{GASTRONOMY AS A TRAVELING MOTIVATING AGENT}

\author{
Susana Suárez Reyes*, Diego Gustavo Ramón Molina**, \\ Edrei del Carmen Izquierdo Alejandro***
}

\begin{abstract}
*Licenciada en Administración. Estudiante de la Maestría en Administración y Dirección Estratégica en la Universidad Juárez Autónoma de Tabasco.

**Licenciado en Mercadotecnia. Estudiante de la Maestría en Administración y Dirección Estratégica en la Universidad Juárez Autónoma de Tabasco. Email: diego_gustavo_nike@hotmail.com

${ }^{* * * E s t u d i a n t e ~ d e ~ l a ~ L i c e n c i a t u r a ~ e n ~ C o n t a d u r i ́ a ~ P u ́ b l i c a ~ e n ~ l a ~ U n i v e r s i d a d ~ J u a ́ r e z ~ A u t o ́ n o m a ~ d e ~ T a b a s c o . ~}$ Email: edrei.izquierdo96@gmail.com
\end{abstract}

Dirección para recibir correspondencia: susy_2793@hotmail.com 


\section{RESUMEN}

OBJETIVO: Identificar las rutas turísticas del Estado de Tabasco, así como el conocimiento que tienen los habitantes del estado acerca de las mismas, con la finalidad de conocer si la gastronomía turística es un factor influyente para realizar un viaje a otro destino o si sólo forma parte secundaria en la elección de viaje de los tabasqueños.

MATERIAL Y MÉTODO: El método utilizado en la investigación tiene un enfoque cuantitativo, de carácter descriptivo y con diseño no experimental, ya que únicamente se pretendió conocer si los tabasqueños conocen las rutas gastronómicas del Estado y el motivo que los mueve a viajar a otro destino.

RESULTADOS: Las personas viajan a otro destino para conocer sus atracciones turísticas, y los tabasqueños no son la excepción, pues el $64 \%$ de los encuestados concuerda en que viajan a otro lugar sólo para conocer sus lugares turísticos y no motivados por la gastronomía del lugar.

CONCLUSIONES: El Estado de Tabasco es muy rico en cultura, naturaleza y gastronomía, el cual dentro de los últimos años ha tenido mayor reconocimiento gracias a varios factores, por lo que el Estado de Tabasco es visto como un lugar atractivo para el desarrollo del turismo gastronómico que puede crear un alto impacto en la sociedad.

PALABRAS CLAVE: Gastronomía. Agente motivador. Rutas turísticas.

\section{ABSTRACT}

OBJECTIVE: To identify the tourist routes of the State of Tabasco, as well as the knowledge that the inhabitants of the state have about them, in order to know if the tourist gastronomy is an influential factor to make a trip to another destination or if it only represents an alternate choice when the inhabitants of Tabasco choose a travel spot.

MATERIAL AND METHOD: The method used in the research has a quantitative, descriptive and non-experimental design, since it was only intended to know if people from Tabasco know the gastronomic routes of the state and the reason that motivates them to travel to another destination. 


\section{LA GASTRONOMÍA COMO AGENTE MOTIVADOR DE VIAJE}

RESULTS: People travel to another destination to know its tourist attractions, and Tabasco is no exception, as $64 \%$ of respondents agree that they travel to another place only to know its tourist places and not motivated by the local cuisine.

CONCLUSIONS: The state of Tabasco is very rich in culture, nature and gastronomy, which in recent years has had greater recognition thanks to several factors, so the state of Tabasco is seen as an attractive place for the development of gastronomic tourism which can generate a high impact on the society.

KEYWORDS: Gastronomy. Motivating agent. Tourist routes.

\section{INTRODUCCIÓN}

La gastronomía siempre ha formado una parte esencial de los viajes por un simple hecho: es una necesidad de orden biológico. Mejail y Vita (2008), mencionan que el turismo cultural ha crecido en los últimos años y dentro de este se ha incorporado el turismo gastronómico, donde las personas, fuera de su lugar de residencia, esperan un servicio diferente al que tienen habitualmente.

Cada lugar cuenta con lugares turísticos y platillos de acuerdo a la región, de los cuales, el Estado de Tabasco es un lugar que cuenta con riquezas naturales y culturales, donde se encuentran rutas turísticas a lo largo de su territorio, en las cuales se pueden realizar distintas actividades.

Dos de estas rutas turísticas están dedicadas a la gastronomía regional, en donde se pueden disfrutar de los diferentes platillos tradicionales de la zona, así como participar en la elaboración de algunos productos gastronómicos.

En definitiva, la gastronomía no es sólo la elaboración de platillos, sino que es toda una manifestación cultural culinaria en la cual se resume la historia, las costumbres, las creencias y los significados del acto de comer (Díaz, 2011). 


\section{Gastronomía turística}

Hoy en día, la gastronomía se ha convertido en uno de los principales motivos para viajar a otro destino, ya sea nacional o internacional, logrando convertir el lugar en un atractivo turístico gastronómico, pues permite conocer otras culturas a través de los alimentos que se degustan.

Los turistas se pueden clasificar en dos tipos, los que "se alimentan" y los que "viajan para comer". Los primeros, en su viaje solamente se alimentan para satisfacer sus necesidades, no es la comida lo que los motiva a viajar. Por otra parte, los segundos realizan un viaje motivados en encontrar placer a través de la gastronomía, siendo ésta el recurso primario del destino visitado (Mazón, Colmenares y Hurtado, 2012).

El recurso gastronómico, frente a otros posibles elementos de la oferta turística local, mantiene la ventaja de poder contar con un producto tangible, sensorial y capaz de comunicar el patrimonio cultural inmaterial escondido detrás de los hábitos culinarios (Di, Hernández y López, 2014).

Para López y Sánchez (2012), el turismo gastronómico se define como uno de los factores mas grandes para potencializar los destinos turísticos, esto debido a que cada vez son mas las personas que viajan motivadas para degustar un platillo de una región o simplemente asistir a un restaurante de una zona geográfica en específico.

Para Vázquez y Agudo (2010), el turismo gastronómico incluye la visita a productores primarios y secundarios de alimentos, festivales gastronómicos, restaurantes y lugares específicos donde la degustación de los atributos de una región especializada en la producción de alimentos es la razón principal para la realización del viaje.

La literatura científica apunta que la gastronomía establece una sinergia con el turismo a través de cuatro aspectos diferentes, pero relacionados entre sí (Tikkanem, 2007, citado por López y Margarida, 2011), (ver tabla 1). 
Tabla 1

Sinergia de la gastronomía y el turismo

\begin{tabular}{ll}
\hline \multicolumn{1}{c}{ Aspecto } & \multicolumn{2}{c}{ Relación con el turismo } \\
\hline Atracción & El destino puede utilizar este elemento para \\
& promocionar dicho lugar. \\
Componente del producto & Se ahonda en el diseño de rutas gastronómicas. \\
Experiencia & La gastronomía adquiere un nivel diferente y se \\
& convierte en un reclamo en sí mismo, siendo un ejemplo \\
& de ello la cocina realizada por los grandes maestros. \\
Fenómeno cultural & Se sustenta en la existencia de diferentes festivales \\
& gastronómicos. \\
\hline
\end{tabular}

Fuente: Elaboración propia (Tikkanem, 2007, citado por López y Margarida, 2011).

Mejail y Vita (2008), mencionan que la gastronomía o la alimentación representa uno de los pilares más importantes sobre los que se sustenta el sector turístico, por lo que se debe prestar más interés al turismo gastronómico, pues es un medio para recuperar la alimentación tradicional de cada zona y es un instrumento que se está convirtiendo en una pieza clave para lograr el posicionamiento de los mismos (Vázquez y Agudo, 2010).

\section{Rutas gastronómicas}

Arriola (2015), menciona que la ruta gastronómica es una variante del turismo y que su función principal es el descubrimiento organizado de un territorio con un enfoque relacionado a los alimentos ya sean locales o regionales.

Por su parte, Jeambey (2015), menciona que las rutas gastronómicas son itinerarios turísticos basados en alimentos que permiten al visitante explorar la cadena de valor del alimento, desde la producción primaria hasta el plato. Además, el autor menciona que existen tres tipos de rutas:

- Rutas que destacan un cierto plato.

- Rutas que destacan una cocina de carácter étnico.

- Rutas que destacan un cierto producto, ya sea alimento primario o elaborado. 


\section{LA GASTRONOMÍA COMO AGENTE MOTIVADOR DE VIAJE}

Así pues, las rutas gastronómicas se integran por productores que reciben a los turistas en sus establecimientos y que les brindan servicios de alimentación, al igual que los restaurantes regionales que privilegian en sus menús platillos tradicionales basados en la producción primaria local y las agroindustrias de la zona (Vázquez y Agudo, 2010).

Las rutas gastronómicas se organizan en torno a un producto clave que caracteriza la ruta y le otorga su nombre, la cual debe ofrecer a quienes la recorren una serie de placeres y actividades relacionadas con los elementos distintivos de la misma: comida, producción agrícola, actividades rurales, entretenimientos en la naturaleza y actividades propias de la cultura regional (Hernández, 2015).

\section{Rutas turísticas gastronómicas del Estado de Tabasco}

Debido al boom petrolero que se originó en el Estado, se generó un notable crecimiento en la infraestructura productiva y urbana, asimismo es considerado hoy en día como un paso obligatorio para acceder al sureste mexicano gracias a su comercio, servicios y turismo (Guzmán y Mayo, 2016).

El Estado de Tabasco tiene un alto potencial turístico, gracias a los atractivos culturales y naturales que tiene en los 17 municipios que lo integran, además de algunos productos gastronómicos realizados en la entidad, también cuenta con 8 rutas turísticas distribuidas alrededor de sus 17 municipios (ver tabla 2).

\section{Tabla 2}

Rutas turísticas del Estado de Tabasco

\begin{tabular}{lll}
\hline \multicolumn{1}{c}{ Ruta Turística } & Municipio & \multicolumn{2}{c}{ Atractivos Turísticos } \\
\hline $\begin{array}{l}\text { La Ruta del Mundo } \\
\text { Maya }\end{array}$ & Balancán & $\begin{array}{l}\text { Zona arqueológica que se compone de diferentes } \\
\text { conjuntos monumentales que incluyen diversos } \\
\text { basamentos y un monumental juego de pelota. }\end{array}$ \\
& Principales atractivos de la capital del estado como: \\
& Yumka, Parque Tomás Garrido Canabal, Corredor \\
La Ruta & Turístico "Paseo Tabasco Malecón", Barco Capitán Beuló \\
Villahermosa & II, Centro Histórico, Parque Tabasco (recinto ferial), \\
& Museo Interactivo Papagayo y el Desarrollo ecoturístico \\
& "La Finca".
\end{tabular}


La Ruta de los

Pantanos

Corredor Biji

Yokot'an

Ruta Ríos

Ruta del Chocolate

Ruta Olmeca

Zoque

Ruta Aventura en

la Sierra
Centla y Jonuta

Nacajuca y

Jalpa de

Méndez

Balancán,

Emiliano Zapata

y Tenosique

Comalcalco, Jalpa de

Méndez,

Paraíso y

Cunduacán

Cárdenas y

Huimanguillo

Jalapa, Teapa,

Tacotalpa y

Macuspana
Reserva de la Biosfera Pantanos de Centla, muelle "Tres

Brazos", playas: Pico de oro, Playa Azul, Miramar y el Bosque, entre otros.

A lo largo de este recorrido se puede disfrutar de la gastronomía típica de la región, los platillos prehispánicos y el pejelagarto son el principal atractivo del tour.

Cascadas de Reforma, Playones del Usumacinta, Zona

Arqueológica de Pomoná, entre otros.

Hacienda "La Chonita", Hacienda "La Luz", Hacienda “Jesús María”, DRUPA, Butifarras, entre otros.

Fuente: Elaboración propia (Buen Viaje, 2017).

El Estado de Tabasco es rico en diversidad cultural y natural; sin embargo, desde hace un tiempo, ha ido destacando no sólo por su atractivo turístico, sino también por sus rutas gastronómicas: la Ruta del Chocolate y el Corredor Biji Yokot'an, además de las actividades que realiza el Estado.

Biji Yokot'an es considerado el corredor gastronómico de Tabasco, famoso por sus restaurantes típicos que ofrecen al público, toda clase de pescado de agua dulce frito o guisado a la tradicional usanza tabasqueña (Buen Viaje, 2017).

Éste corredor turístico abarca los municipios de Nacajuca y Jalpa de Méndez y se puede disfrutar de la gastronomía típica de la región; los platillos prehispánicos y el pejelagarto, son dos de los platillos principales y más atractivos de éste corredor. 


\section{LA GASTRONOMÍA COMO AGENTE MOTIVADOR DE VIAJE}

Por su parte, la Ruta del Chocolate como producto turístico puede ser atractivo, pero conocer de cerca el fruto, ver su procesamiento, olerlo y saborearlo es más atractivo, sumándose la belleza natural y el clima de la entidad que le dan singular sabor (Buen Viaje, 2017).

En la Ruta del Cacao, se lleva a cabo un recorrido por las haciendas chocolateras de la entidad en el municipio de Comalcalco, en las cuales se puede participar en la elaboración del producto, así como el museo interactivo del chocolate DRUPA, en el municipio de Cunduacán. De igual manera, en el municipio de Jalpa de Méndez se disfruta de la gastronomía local y sin duda alguna, las famosas butifarras son la principal atracción de este municipio (ver tabla 3).

\section{Tabla 3}

\section{Actividades gastronómicas del Estado de Tabasco}

\begin{tabular}{|c|c|}
\hline Municipio & Actividad \\
\hline Comalcalco & $\begin{array}{ll}\text { - } & \text { Museo del chocolate } \\
\text { - } & \text { Hacienda La Luz } \\
\text { - } & \text { Hacienda La Chonita } \\
\text { - } & \text { Hacienda Jesús María } \\
\text { - } & \text { Taller de producción y cultivo del cacao criollo } \\
\text { - } & \text { Taller de procesos de fermentado y secado } \\
\text { - } & \text { Taller de industrialización del chocolate } \\
\text { - } & \text { Taller de procesos de fabricación de chocolates y derivados del cacao } \\
\text { - } & \text { Taller y demostración del proceso del chocolate CACEP }\end{array}$ \\
\hline Centro & $\begin{array}{l}\text { - } \\
\text { - } \\
\text { Paletas La Polar } \\
\text { - } \\
\text { Festival del Pozol }\end{array}$ \\
\hline Tenosique & $\begin{array}{ll}\text { - } & \text { Festival del queso } \\
\text { - } & \text { Quesos Don Rodo }\end{array}$ \\
\hline Jalpa de Méndez & - $\quad$ Butifarra \\
\hline Tacotalpa & - Restaurante el Sabor de la Sierra \\
\hline Paraíso & - Diversos restaurantes que ofrecen platillos derivados del marisco. \\
\hline Cunduacán & - Museo Interactivo del Chocolate DRUPA \\
\hline
\end{tabular}

Fuente: Elaboración propia (Rutas Gastronómicas, s.f.) 


\section{MATERIAL Y MÉTODO}

\section{Diseño de la investigación}

El propósito de la investigación fue descriptivo y con diseño no experimental (Hernández, Fernández y Baptista, 2010), ya que únicamente se dio a conocer si los tabasqueños conocen las rutas gastronómicas de su Estado y el motivo que los mueve a viajar a otro destino. El estudio es de tipo cuantitativo apoyado de un sondeo.

\section{Muestra}

Se realizó un muestreo a los residentes del Estado de Tabasco, específicamente a las personas mayores de 18 años, a las cuales se realizó un sondeo exprés.

El objetivo principal fue determinar las motivaciones a la hora de viajar y sus conocimientos acerca de rutas gastronómicas en el Estado de Tabasco, para tener así un panorama general acerca del turismo gastronómico.

El tipo de muestra fue no probabilística, por lo cual se aplicó el sondeo a 100 personas al azar, que cumpliera con el requisito previamente mencionado.

\section{Técnica de investigación}

Se llevó a cabo una investigación en fuentes secundarias, apoyada de un sondeo, puesto que se requería recolectar información sobre el conocimiento que tienen los tabasqueños sobre las rutas turísticas gastronómicas del Estado, así como su principal agente motivador de viaje.

\section{Instrumento}

Para la recolección de datos, se realizó un sondeo en el cual se solicitó información a través de las siguientes variables:

- Género.

- Edad.

- Motivación del viaje.

- Conocimiento de las rutas turísticas gastronómicas del Estado de Tabasco. 


\section{LA GASTRONOMÍA COMO AGENTE MOTIVADOR DE VIAJE}

\section{RESULTADOS}

A continuación, se muestran los resultados obtenidos en el sondeo realizado a 100 personas del Estado de Tabasco.

De acuerdo a los resultados obtenidos, el $45 \%$ de las personas encuestadas son hombres, mientras que el $55 \%$ son mujeres.

\section{Tabla 4}

Distribución de frecuencias por género

\begin{tabular}{lcccc}
\hline & Frecuencia & Porcentaje & Porcentaje válido & Porcentaje acumulado \\
\hline Hombre & 45 & 45.0 & 45.0 & 45.0 \\
Mujer & 55 & 55.0 & 55.0 & 100.0 \\
Total & 100 & 100.0 & 100.0 & \\
\hline
\end{tabular}

Fuente: Elaboración propia.

La edad más joven encuestada fue de 18 años, mientras que la persona más grande fue de 39 años (ver tabla 5).

La mayoría de los encuestados se encuentran en el intervalo de edad de 18 a 24 años (70\%), mientras que en el rango de 32 a 39 años se encuentra la menor cantidad de personas encuestadas (5\%).

Tabla 5

Distribución de frecuencias por rango de edad

\begin{tabular}{lcccc}
\hline & Frecuencia & Porcentaje & Porcentaje válido & Porcentaje acumulado \\
\hline 18 a 24 & 70 & 70.0 & 70.0 & 70.0 \\
25 a 31 & 25 & 25.0 & 25.0 & 95.0 \\
32 a 39 & 5 & 5.0 & 5.0 & 100.0 \\
Total & 100 & 100.0 & 100.0 & \\
\hline
\end{tabular}

Fuente: Elaboración propia.

Por lo general, las personas viajan a otro destino para conocer sus atracciones turísticas, y los tabasqueños no son la excepción, pues el $64 \%$ de los encuestados concuerda en que viajan a SUÁREZ-REYES S., RAMÓN-MOLINA D. G., IZQUIEDO-ALEJANDRO E. DEL C. 


\section{LA GASTRONOMÍA COMO AGENTE MOTIVADOR DE VIAJE}

otro lugar sólo para conocer sus lugares turísticos y no motivados por la gastronomía del lugar, que como puede verse en la tabla 6 , sólo el $9 \%$ lo hace motivado por ésta razón.

\section{Tabla 6}

¿Cuando viajas a otro destino, lo haces atraído por el lugar o para probar la gastronomía tradicional del mismo?

\begin{tabular}{lcccc}
\hline & Frecuencia & Porcentaje & Porcentaje válido & Porcentaje acumulado \\
\hline Turismo & 64 & 64.0 & 64.0 & 64.0 \\
Gastronomía & 9 & 9.0 & 9.0 & 73.0 \\
Ambas & 27 & 27.0 & 27.0 & 100.0 \\
Total & 100 & 100.0 & 100.0 & \\
\hline
\end{tabular}

Fuente: Elaboración propia.

El Estado de Tabasco cuenta con dos rutas turísticas gastronómicas, de las cuales sólo el 55\% de los encuestados saben de ellas, mientras que el $45 \%$ no tenían idea de que éstas existían en la entidad. Esto representa un indicador grave, pues el porcentaje de las personas que desconocen los lugares turísticos gastronómicos del Estado es alto. Esto no debería ser así, pues al ser residentes tabasqueños lo ideal sería que conocieran acerca de su Estado.

\section{Tabla 7}

¿Sabes cuáles son las rutas turísticas gastronómicas del Estado de Tabasco?

\begin{tabular}{lcccc}
\hline & Frecuencia & Porcentaje & Porcentaje válido & $\begin{array}{c}\text { Porcentaje } \\
\text { acumulado }\end{array}$ \\
\hline $\mathrm{Si}$ & 55 & 55.0 & 55.0 & 55.0 \\
$\mathrm{No}$ & 45 & 45.0 & 45.0 & 100.0 \\
Total & 100 & 100.0 & 100.0 & \\
\hline
\end{tabular}

Fuente: Elaboración propia.

Al momento de entrevistar a las personas, muchas de las que no tenían conocimiento de la existencia de éstas rutas gastronómicas mencionaban que el Estado no hace mucha difusión SUÁREZ-REYES S., RAMÓN-MOLINA D. G., IZQUIEDO-ALEJANDRO E. DEL C. 


\section{LA GASTRONOMÍA COMO AGENTE MOTIVADOR DE VIAJE}

sobre las mismas, sino que le dan mayor difusión a las rutas turísticas como los museos y zonas arqueológicas, pero no a las actividades gastronómicas.

\section{CONCLUSIONES}

El Estado es muy rico en cultura, naturaleza y gastronomía y dentro de los últimos años ha tenido mayor reconocimiento gracias a éstos factores.

Actualmente, se concibe como un escenario atractivo para implementar acciones que propicien el desarrollo del turismo de negocios a gran escala y con un alto impacto, capaz de convertirse en un destino turístico de referencia inevitable tanto para el contexto nacional como internacional (Mayo, Guzmán y Luna, 2016).

Se puede concluir que los turistas viajan por dos motivos: para conocer otros lugares y para conocer el destino a través de su gastronomía; sin embargo, la mayor parte de los turistas viajan atraídos por el lugar, no por la gastronomía tradicional que se les pueda ofrecer.

Es aquí donde se deben utilizar las rutas gastronómicas como estrategia para fortalecer el sector y, lo más importante, dar a conocer todos los destinos turísticos y la gastronomía local y/o regional de un estado; las rutas turísticas no deben ser vistas como una estrategia de promoción si no como una fuente de productividad e ingresos para la región (Arriola, 2015).

El Estado de Tabasco cuenta con amplios lugares turísticos y gastronomía tradicional a lo largo de todo su territorio, motivo por lo cual se crean las rutas turísticas, dos de ellas dedicadas a su gastronomía: la Ruta del Chocolate y el corredor turístico Biji Yokot'an, donde puede disfrutarse de la gastronomía del Estado, además de los talleres de elaboración del producto. Sin embargo, cerca del $50 \%$ de los encuestados tabasqueños no tenían noción de su existencia.

En definitiva, el Estado debería hacer mayor difusión de las rutas gastronómicas con que cuenta Tabasco, pues al momento de realizar el sondeo las personas mencionaron que se le ha prestado mayor atención a las zonas turísticas del mismo, como las zonas arqueológicas, museos y pueblos mágicos, pero hay muy poca publicidad acerca de la gastronomía con la que cuenta Tabasco. 
LA GASTRONOMÍA COMO AGENTE MOTIVADOR DE VIAJE

\section{REFERENCIAS BIBLIOGRÁFICAS}

Arriola, A. (2015). Las rutas gastronómicas como estrategia de desarrollo turístico local. Research Gate. Recuperado de https://www.researchgate.net/publication/282133780

Buen viaje. (2017). Rutas turísticas de Tabasco. Una buena forma de conocer todo el estado. Recuperado de http://www.revistabuenviaje.com/conocemexico/destinos/tabasco/rutastabasco/rutasdetaba sco.php

Di, E., Hernández, J.M. y López, T. (2014). La gastronomía como patrimonio cultural y motor del desarrollo turístico. Un análisis DAFO para Extremadura. Monográfico, (9), 817-833.

Díaz, M.M. (2011). La gastronomía de México. Identidad cultural desvalorada en el ámbito del turismo mexicano (Tesis de maestría, Universidad de Girona, Catalunya). Recuperada de http://dugidoc.udg.edu/bitstream/handle/10256/10111/DiazRamosMonicaMargarita_Treball. pdf?sequence $=1$

Guzmán, A. y Mayo, A. (2016). Evaluación de los atractivos turísticos de Tabasco, México. International Journal of Scientific Managment Tourism, 2(2), 151-161.

Hernández, R., Fernández, C. y Baptista, P. (2010). Metodología de la investigación. México: McGraw-Hill.

Hernández, R.D. (2015). Gestión y planificación de rutas turísticas gastronómicas: estudio de caso en la provincia de Córdoba (Tesis doctoral, Universidad de Córdoba). Recuperada de http://helvia.uco.es/xmlui/bitstream/handle/10396/13107/2015000001211.pdf?sequence=1

Jeambey, Z. (2015). Rutas Gastronómicas y Desarrollo local: un ensayo de conceptualización en Cataluña. PASOS, 14(5), 1187-1198.

López, T. y Margarida, M. (2011). Turismo, cultura y gastronomía. Una aproximación a las rutas culinarias. International conference on tourism \& management studies, (1), 916-922.

López, T. y Sánchez, S. M. (2012). La gastronomía como motivación para viajar. Un estudio sobre el turismo culinario en Córdoba. PASOS, 10(5), 575-584. 
Mayo, A., Guzmán, A. y Luna, L.I. (2016). Tabasco en la necesaria convergencia de sus proyectos de inversión en el turismo de negocios y de ocio. International Journal of Scientific Management and Tourism, 2(1), 355-370.

Mazón, T., Colmenares, M. y Hurtado, J.A. (2012). Turismo gastronómico y turismo de masas: la satisfacción de los turistas con la alimentación que reciben en Benidorm. Gran Tour: Revista de Investigaciones Turísticas, (6), 122-141.

Mejail, A. y Vita, M. (2008). La gastronomía como atractivo turístico y factor de desarrollo. Escuela de economía y negocios. (1), pp. 1-58. Recuperado de http://www.unsam.edu.ar/escuelas/economia/CIDeTur/boletines/Estudio\%20Tom\%C3\%A1 s\%20Jofr\%C3\%A9\%20-Parte\%201.pdf

Rutas Gastronómicas. (s.f.). Tabasco en la Ruta del Cacao. Recuperado de www.rutasgastronomicas. $\mathrm{mx}$

Vázquez, M.G. y Agudo, E.M. (2010). El turismo gastronómico y las Denominaciones de origen en el sur de España: Oleoturismo. Un estudio de caso. Pasos, 8(1), 91-112. 